\title{
Etude Eco-Dendrométrique Du Dépérissement Du Cèdre De l'Atlas Dans Le Parc National De Theniet El Had "Algérie"
}

\section{Taleb Mohamed Lamine, MA}

Centre universitaire de Tissemsilt, Algérie

Maatoug M'hamed, Prof

Laboratoire d'Agro Biotechnologie et de Nutrition en Zones Semi Arides, Faculté Des Sciences de la Nature et de la Vie. Université de Tiaret, Algérie Azouzi Blel, Prof

Université de Djelfa, Algérie

\section{Zedek Mohamed, MA}

Université de Tiaret, Algérie

Hellal Benchabane, Prof

Université de Sidi bel abbas, Algérie

doi: 10.19044/esj.2016.v12n29p112 URL:http://dx.doi.org/10.19044/esj.2016.v12n29p112

\begin{abstract}
This study aims to search the relationship between the decline of the Atlas cedar and the eco-dendrometrique factors in the National Park of Theniet El Had located in the north-west of Algeria. This study takes place throughout 30 circular plots of $1.000 \mathrm{~m}^{2}$ area in which, a dendrometric measures and ecological data are taken in addition to descriptive data for Atlas cedar trees.

The descriptive data shows that $34 \%$ of inventoried Atlas cedar have damaged leaves and $30 \%$ have more then $25 \%$ of their crowns damaged.

The analysis of variance shows that there is no relationship between the rate of the Atlas cedar decline ant the ecological factors, components of the soil and dendrometric parameters except for the average circumference witch is influenced by the competition between trees.

Therefor, a particular management plan for the regulation of competition is a necessity for this park.
\end{abstract}

Keywords: Eco-dendrometric factors, decline, Atlas cedar, Theniet el had national park 


\section{Introduction}

En Algérie, les peuplements du cèdre de l'Atlas occupent une surface d'environ 30000 ha, ils sont localisés au centre et à l'Est, où ils forment de belles futaies.

Plusieurs cédraies de vaste étendue ont été classées en qualité de parcs nationaux, avec un statut de gestion scientifique (Haddar, 2007).

Quoique géré en parc national, le cèdre reste soumis à plusieurs contraintes à savoir « le pâturage, les coupes illicites et la pollution » ainsi que le phénomène de dépérissement qui constitue un fléau majeur menaçant la pérennité des cédraies.

La cédraie de Theniet El Had, longtemps admirée par sa beauté naturelle (Boudy, 1950) est le premier parc national créé en Algérie pendant la période coloniale soit en 1923 et reproclamé encore parc national après l'indépendance en 1983. Il a connu le phénomène de dépérissement en 1984 où le service forestier signala 3000 arbres de cèdre dépéris. Cette situation a mené les scientifiques à rechercher d'éventuelles causalités de ce fléau complexe où plusieurs hypothèses ont été révélées «changements climatiques, attaques parasitaires, pollution, etc. » sans qu'aucune d'elles ne soit confirmée.

A cet effet, et dans le cadre de la réalisation d'un travail descriptif du dépérissement du cèdre, l'identification de l'état sanitaire des peuplements dépéris et l'étude de la relation entre ce dépérissement et les paramètres dendrométriques, stationnels et édaphiques via l'analyse de la variance à un facteur au seuil de signification de 95\% fera l'objet de cette étude.

\section{Matériel et méthodes}

\section{Présentation de la zone d'étude}

La zone d'étude correspond au versant nord du parc national de Theniet El Had relevant de la wilaya de Tissemsilt,

Le parc national recouvre les deux versants de Djebel El Meddad, partie prenante du grand massif de l'Ouarsenis constituant, ensemble, la chaine sud de l'Atlas tellien. Il s'étale sur une superficie de 3424 ha dont 2968 ha couverte de végétaux comprise entre intervalle altitudinale allant de $858 \mathrm{~m}$ à $1787 \mathrm{~m}$.

Il est soumis à l'étage bioclimatique Subhumide à hiver froid avec une période sèche de quatre mois du début juin jusqu’à la mi-octobre.

\section{Méthodologie}

$\mathrm{Vu}$ la facilité de délimitation et l'absence de direction privilégiée (Palm, 1977 ; Parde et Bouchon, 1988), Au total, trente placettes circulaires d'une superficie de 10 ares y sont installées au sein des taches du 
dépérissement à travers le versant nord du parc, particulièrement, dans les cantons de Guerouaou, Djouareb, Rond-point, Pré-Benchohra et Pépinière (Figure 1).

Chaque placette a fait l'objet de mesures dendrométriques et la récolte des données stationnelles à savoir :

- $\quad$ Données dendrométriques : Circonférence à 1,30 m, hauteur totale, pour toutes les espèces, en plus, hauteur du houppier, hauteur jusqu'à la base du houppier, hauteur de la cime morte pour les arbres de cèdre.

- Données stationnelles : altitude, exposition, pente, microrelief et propriétés physico-chimiques du sol. Celles-ci étant déterminées sur la base de prélèvement d'échantillons par le biais de la tarière pédologique. A raison d'un relevé par placette, chaque échantillon est prélevé au niveau de l'horizon $A_{1}$, soit celui qualifié d'horizon organo-minéral du fait qu'il renferme les éléments organiques et minéraux (Riou-nivert, 2005 ; Drénou, 2006).

Par ailleurs, l'état de santé des tiges de cèdre, retenu d'ordre symptomatologique, est identifié par le biais des caractères suivants : maladies du feuillage et évaluation des dégâts du houppier par tiers.

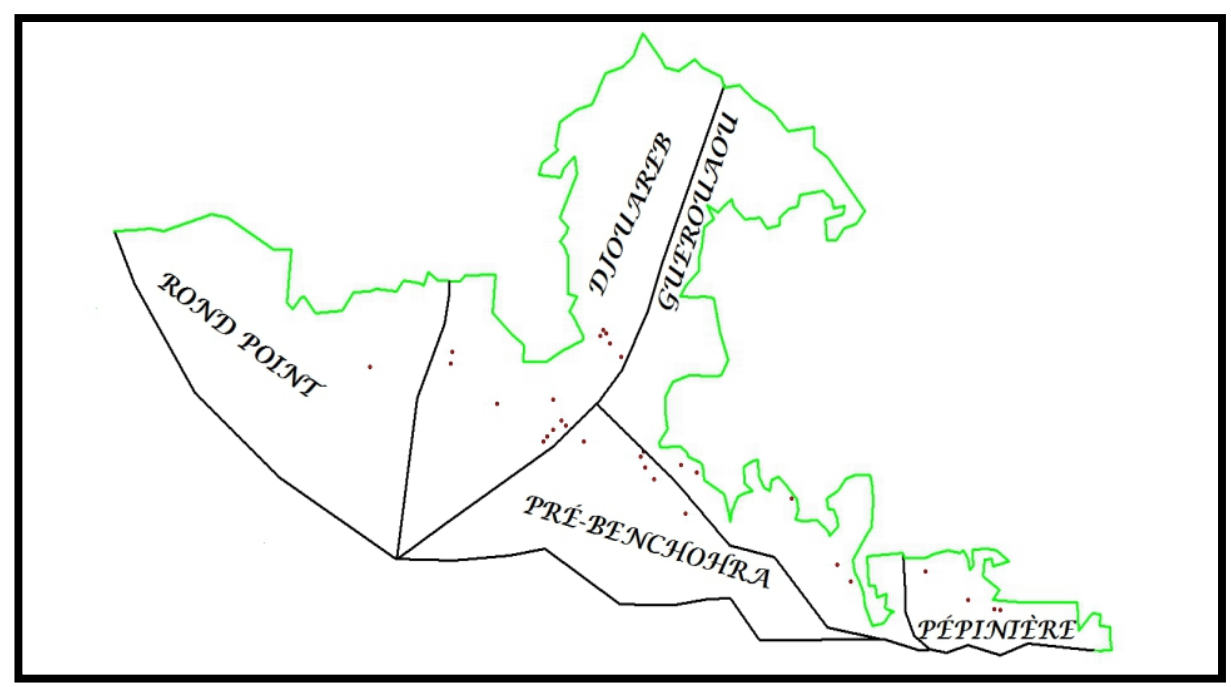

Figure 1 : Répartition des placettes d'étude

\section{Résultats et discussions}

Les peuplements échantillonnés présentent des mélanges de chêne vert, de chêne zeen, et à une présence moindre le chêne liège et le genévrier oxycèdre avec comme espèce principale, le cèdre de l'Atlas. 


\section{Santé des peuplements de cèdre}

\section{Maladie du feuillage}

La vigueur des arbres est régie par la vitalité de leur feuillage (abondance et couleur). Afin de décrire le peuplement inventorié, sur la base des situations sanitaires des aiguilles, quatre critères qualitatifs sont mis en évidence: feuillage sain, chute des aiguilles, dommage physique et dépérissement des feuillages (Figure 2).

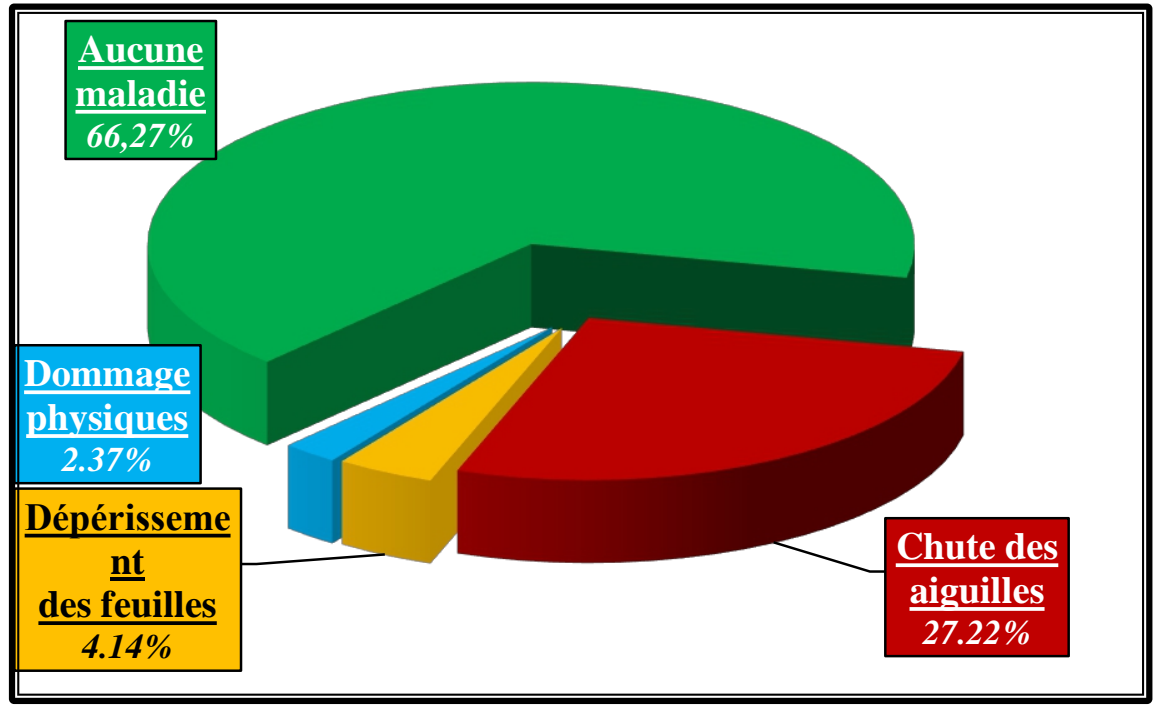

Figure 2 : Distribution en fréquences relatives de l'état de santé du feuillage des peuplements de cèdre recensés

A travers la Figure 2, on relève que $66 \%$ du feuillage présente un bon état sanitaire. La strate relative aux dommages des aiguilles est évaluée à $34 \%$ de l'ensemble des tiges inventoriées. Cette situation ne peut que signifier un souci de dégradation d'une ampleur.

\section{Etat sanitaire du houppier}

Le houppier d'un arbre est l'ensemble des branches et des rameaux qui entourent la partie supérieure de l'arbre (Rondeux, 1993). L'état sanitaire du houppier d'un arbre est fortement corrélé avec sa croissance. Un houppier endommagé n'est autre qu'un indicateur de souffrance d'un arbre.

L'appréciation du déficit foliaire s'est effectuée par l'évaluation de la fréquence de dégradation du houppier tel que le démontre le Tableau 1 (O.N.F, 2010) : 
Tableau 1 : Classe de notation des dégâts des houppiers

\begin{tabular}{||c|c||}
\hline Fréquence & Indicatif\% \\
Nulle à très faible & 0 à $5 \%$ \\
Faible & 6 à $25 \%$ \\
Modérée & 26 à $50 \%$ \\
Importante & 51 à $75 \%$ \\
Très importante & 76 à $95 \%$ \\
\hline Total & 96 à $100 \%$ \\
\hline
\end{tabular}

La Figure 3 révèle que plus de 30\% du peuplement recensé présente des dommages sur les houppiers. Indication fort compromettante de sa croissance.

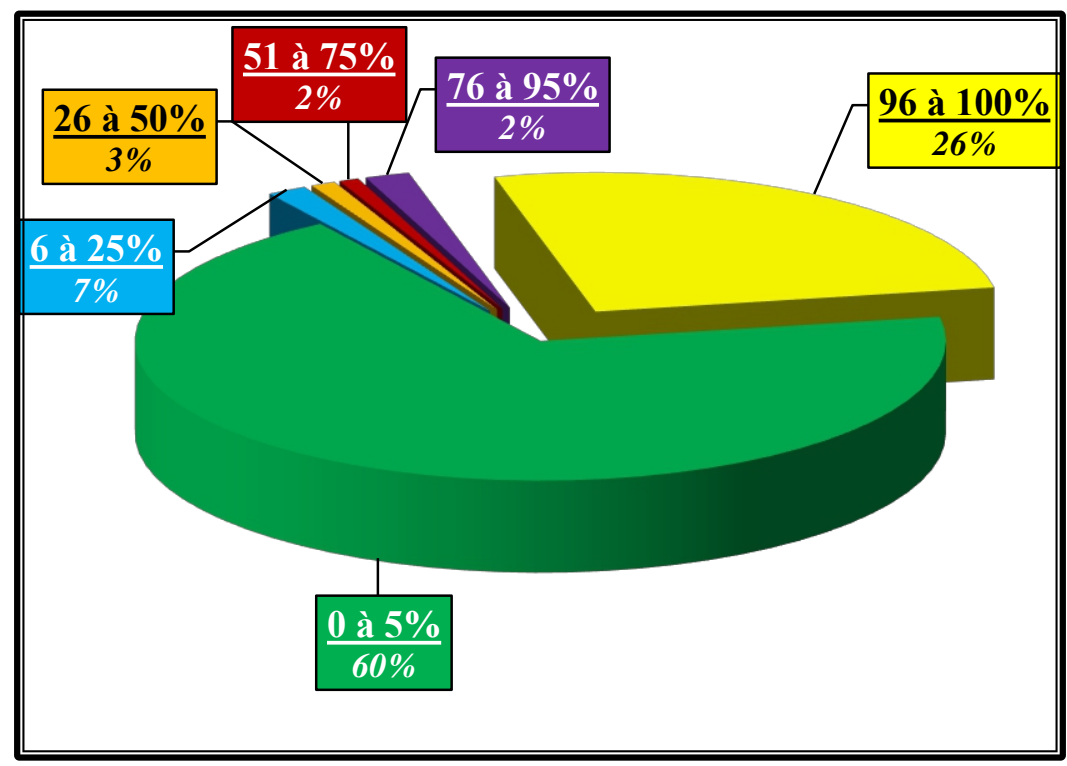

Figure 3 : Distribution en fréquence relative des classes des dégâts du houppier des peuplements de cèdre recensés

\section{Analyse du dépérissement}

L'analyse de la variance à un facteur, au seuil de signification de $95 \%$, est celle qui a fait l'objet d'analyse appropriée quant aux variabilités du taux de dépérissement à travers l'ensemble des strates de chacune des variables précitées. Cette analyse statistique demeure l'outil fiable dans de telle étude (Dagnelie, 1994).

\section{Relation dépérissement-Caractéristiques stationnelles}

\section{a) Relation dépérissement-caractéristiques topographiques}

Les caractéristiques topographiques étudiées sont celles jouant un rôle dans : la rétention de l'eau «Le microrelief », les variations climatiques «L'étagement altitudinal », ruissellement de l'eau «La pente» et la 
pluviométrie «L'exposition » (Guehl, 1984 ; Le goff et Levy, 1984 ; Seigue, 1985 ; Chbouki, 1994)

Le résultat de l'analyse de la variance émet une différence non significative (Tableau 2) ce qui signifie que la causalité du dépérissement ne réfère pas aux caractéristiques topographiques. En effet cette situation est dégagée du fait que la zone d'étude se localise en totalité dans le versant connu par une pluviométrie et une humidité importante à celle du versant sud.

Tableau 2 : Mise en évidence du dépérissement à travers les caractéristiques topographiques

\begin{tabular}{|c|c|c|}
\hline Paramètres topographique & $\overline{F_{\text {observé }}}$ & $F_{\text {théorique }}$ \\
\hline Microrelief & 0.6012212 & 3.3541308 \\
\hline Etagement altitudinal & 0.7640499 & 3.354130829 \\
\hline Pente du terrain & 3.19515903 & 3.35413083 \\
\hline Exposition & 0.260048555 & 4.195971707 \\
\hline
\end{tabular}

\section{b) Relation dépérissement-caractéristiques édaphiques}

Les échantillons du sol prélevés, au nombre de trente, ont fait l'objet d'analyse physico-chimiques au laboratoire pour enfin ressortir le taux de calcaire, le $\mathrm{PH}$, le taux de la matière organique et la texture du sol

Chacune des variables édaphiques a fait l'objet du traitement statistique avec le taux de dépérissement par le biais de l'analyse de la variance.

Il en ressort que le dépérissement n'est significativement corrélé avec aucune de ces variables (Tableau 3).

Tableau 3 : Mise en évidence du dépérissement à travers les caractéristiques édaphiques

\begin{tabular}{|c|c|c|}
\hline Paramètres édaphique & $F_{\text {observé }}$ & $F_{\text {théorique }}$ \\
Taux de calcaire & 1,18136725 & 4,19597171 \\
Texture du sol & 0,82740627 & 3,35413083 \\
Taux de la matière organique & 1,76835819 & 3,35413083 \\
PH du sol & 4,05770725 & 4,19597171 \\
\hline
\end{tabular}

Le taux de calcaire, identifiant l'apport en calcium nécessaire aux végétaux, est retenu comme étant un élément favorisant la stabilité de la structure et la perméabilité des sols (Mathieu et Pieltain, 2003). Suite à la faible variabilité déduite, soit de 0 à $2.09 \%$, le taux de dépérissement ne présente aucune différence significative.

La texture du sol est dans l'ensemble meuble, soit celle appréciée par le cèdre de l'Atlas (Benabid, 1994 ; Ripert et Boisseau, 1994 ; Benmouffok, 1994 ; Lecomte, 2007).

Les valeurs de $\mathrm{pH}$ de sol déduites révèlent une homogénéité traduite par des valeurs variant entre 6.4 et 7 .

La matière organique n'est autre que la décomposition des débris végétaux et animaux. Sa teneur totale dans le sol renseigne sur sa potentialité 
fertilisante (Mathieu et Pieltain, 2003). Ainsi, les échantillons de sols présentent une variabilité de matière organique variant entre $1 \%$ et $4,5 \%$,

Indifférent à la nature lithologique et aux propriétés chimiques du sol, il préfère néanmoins les sols meubles, profonds et caillouteux (Toth, 1970 ; Benabid, 1994 ; Benmouffok, 1994 ; Ripert et Boisseau, 1994 ; Demarteau et al., 2007 ; Lecomte, 2007).

\section{Relation dépérissement-caractéristiques dendrométriques}

\section{a) Identification de la variabilité dendrométrique}

Les variables dendrométriques révèlent être le signe indicateur d'identification de la structure et de l'état de productivité des peuplements forestiers (Rondeux, 1993 ; Rondeux et al., 1995). Dès lors une synthèse sur la variabilité dendrométriques serait d'une utilité importante pour décrire la nature des peuplements échantillonnés (Tableau 3 et 4).

Tableau 4 : Variabilité dendrométriques des espèces inventoriées

\begin{tabular}{|c|c|c|c|c|c|c|}
\hline & & \begin{tabular}{|c|} 
Cèdre \\
de l'Atlas \\
\end{tabular} & $\begin{array}{c}\text { Chêne } \\
\text { vert }\end{array}$ & $\begin{array}{c}\text { Chêne } \\
\text { zeen }\end{array}$ & $\begin{array}{l}\text { Chêne } \\
\text { liège }\end{array}$ & $\begin{array}{l}\text { Genévrier } \\
\text { oxycèdre }\end{array}$ \\
\hline \multirow{3}{*}{$\begin{array}{l}\text { Circonférence } \\
\text { moyenne à } \\
1.30 \mathrm{~m}(\mathrm{~m})\end{array}$} & Minimale & 0.98 & 0.25 & 0.45 & 0.68 & 0.23 \\
\hline & Moyenne & 1.54 & 0.52 & 1.19 & 0.81 & 0.63 \\
\hline & Maximale & 2.12 & 0.95 & 2.07 & 0.94 & 1.01 \\
\hline \multirow{3}{*}{$\begin{array}{l}\text { Hauteur totale } \\
\text { moyenne }(\mathrm{m})\end{array}$} & Minimale & 8.35 & 2.50 & 9 & 3.75 & 3.00 \\
\hline & Moyenne & 16.09 & 4.50 & 13.66 & 4.69 & 3.97 \\
\hline & Maximale & 25.38 & 8.00 & 20.33 & 5.63 & 4.55 \\
\hline \multirow{3}{*}{$\begin{array}{c}\text { Surface } \\
\text { terrière totale } \\
\left(\mathrm{m}^{2} / \mathrm{ha}\right)\end{array}$} & Minimale & 10.40 & 0.10 & 0.16 & 0.37 & 0.04 \\
\hline & Moyenne & 33.46 & 3.83 & 4.52 & 0.89 & 3.45 \\
\hline & Maximale & 71.39 & 9.74 & 9.66 & 1.41 & 6.76 \\
\hline \multirow{3}{*}{$\begin{array}{l}\text { Densité totale } \\
\quad \text { (pieds/ha) }\end{array}$} & Minimale & 60 & 10 & 10 & 10 & 10 \\
\hline & Moyenne & 169 & 146 & 27 & 15 & 78 \\
\hline & Maximale & 420 & 460 & 70 & 20 & 190 \\
\hline
\end{tabular}

Tableau 5: Variabilité dendrométriques des peuplements de cèdre inventoriées

\begin{tabular}{|c|c|c|c|c|c|c|c|c|c|c|c|}
\hline & 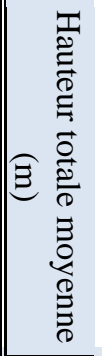 & 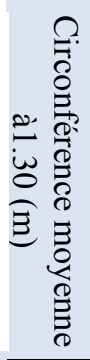 & 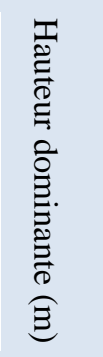 & 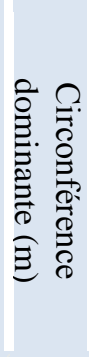 & 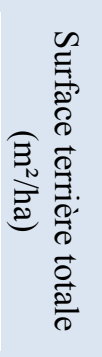 & 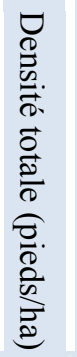 & 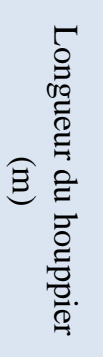 & 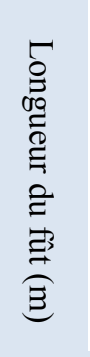 & 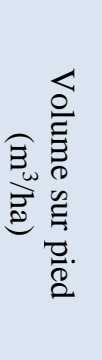 & 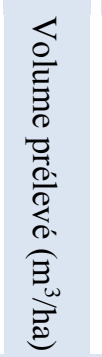 & 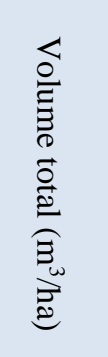 \\
\hline Minimale & 8.35 & 0.98 & 11.37 & 1.31 & 10.40 & 60 & 4.99 & 1.74 & 153.7 & 0.00 & 153.7 \\
\hline Moyenne & 16.09 & 1.54 & 18.98 & 1.83 & 33.46 & 169 & 11.82 & 4.41 & 336.7 & 59.42 & 396.15 \\
\hline Maximale & 25.38 & 2.12 & 27.80 & 2.43 & 71.39 & 420 & 22.13 & 10.81 & 790.5 & 365.6 & 829.7 \\
\hline
\end{tabular}


Le tableau 4 montre que le cèdre de l'Atlas est en mélange. Avec la présence d'une importante densité de chêne vert et très faible densité de chêne liège.

Le tableau 5 montre que les peuplements de cèdre échantillonnés présentent une grande variabilité dendrométrique d'une placette à l'autre.

Cette situation compromettante n'est que la conséquence d'exclusion de toute sortes d'intervention sylvicole dans une cédraie issue naturellement est régie en parc national avec.

L'étude de la relation du dépérissement avec les caractéristiques dendrométrique est basée sur trois axes, selon que la variation dendrométrique influence sur l'état des peuplements échantillonnés, soit celle :

- Variables dendrométriques liées à la fertilité stationnelle « la hauteur dominante et la circonférence dominante »

- Variables dendrométriques liées à la compétition des arbres «la surface terrière totale, la densité totale, la hauteur moyenne et la circonférence moyenne à 1.30 »

\section{b) Relation dépérissement-variables liées à la fertilité}

La circonférence dominante et la hauteur dominante sont de très bons indices de la productivité d'un peuplement liée à l'effet des différents facteurs de production de la station et loin d'être affecté par la concurrence (Decourt, 1967).

L'analyse de la variance, pour la relation dépérissement circonférence dominante et dépérissement hauteur dominante émet un résultat non significatif (Tableau 5) ce qui signifie que le dépérissement est loin d'être corrélé avec la fertilité stationnelle. Ce résultat confirme parfaitement celui de la relation entre le dépérissement et les facteurs stationnels.

Tableau 6 : Mise en évidence du dépérissement à travers les variables liées à la fertilité

\begin{tabular}{|c|c|c|}
\hline Paramètres édaphique & $F_{\text {observé }}$ & $F_{\text {théorique }}$ \\
\hline Circonférence dominante & 0.69406407 & 3.35413083 \\
Hauteur dominante & 0.904722268 & 2.620654147 \\
\hline
\end{tabular}

\section{c) Relation dépérissement-variables liées à la compétition}

La surface terrière totale, la densité totale, et la circonférence moyenne à $1.30 \mathrm{~m}$ sont des variables soumises à l'influence de la concurrence des tiges (Bellefleur et Villeneuve, 1984 ; Deleuze et al., 1996 ; Rondeux, 1993 ; Alteyrac, 2005).

La différence non significative est le résultat obtenu entre le dépérissement et chacune des variables liées à la compétition, par le biais de l'analyse de la variance, à l'exception de la relation dépérissement- 
circonférence moyenne (Tableau 6). Cette situation mène à prédire que le dépérissement est influencé par une variable fort considérablement liée à la compétition entre les tiges voisines. La figure 4 démontre que de plus en plus que les tiges sont grosses et comme elles ne sont soumises à aucune intervention sylvicole, plus le dépérissement augmente, faute d'espace vital compromettant.

Tableau 7 : Mise en évidence du dépérissement à travers les variables liées à la compétition

\begin{tabular}{|c|c|c|}
\hline Paramètres édaphiques & $\mathrm{F}_{\text {observé }}$ & $\mathrm{F}_{\text {théorique }}$ \\
Surface terrière totale & 0.51803910 & 4.19597171 \\
Densité totale & 2.96595333 & 2.97515397 \\
\hline Circonférence moyenne à $1.30 \mathrm{~m}$ & 6.63919311 & 3.35413083 \\
\hline
\end{tabular}

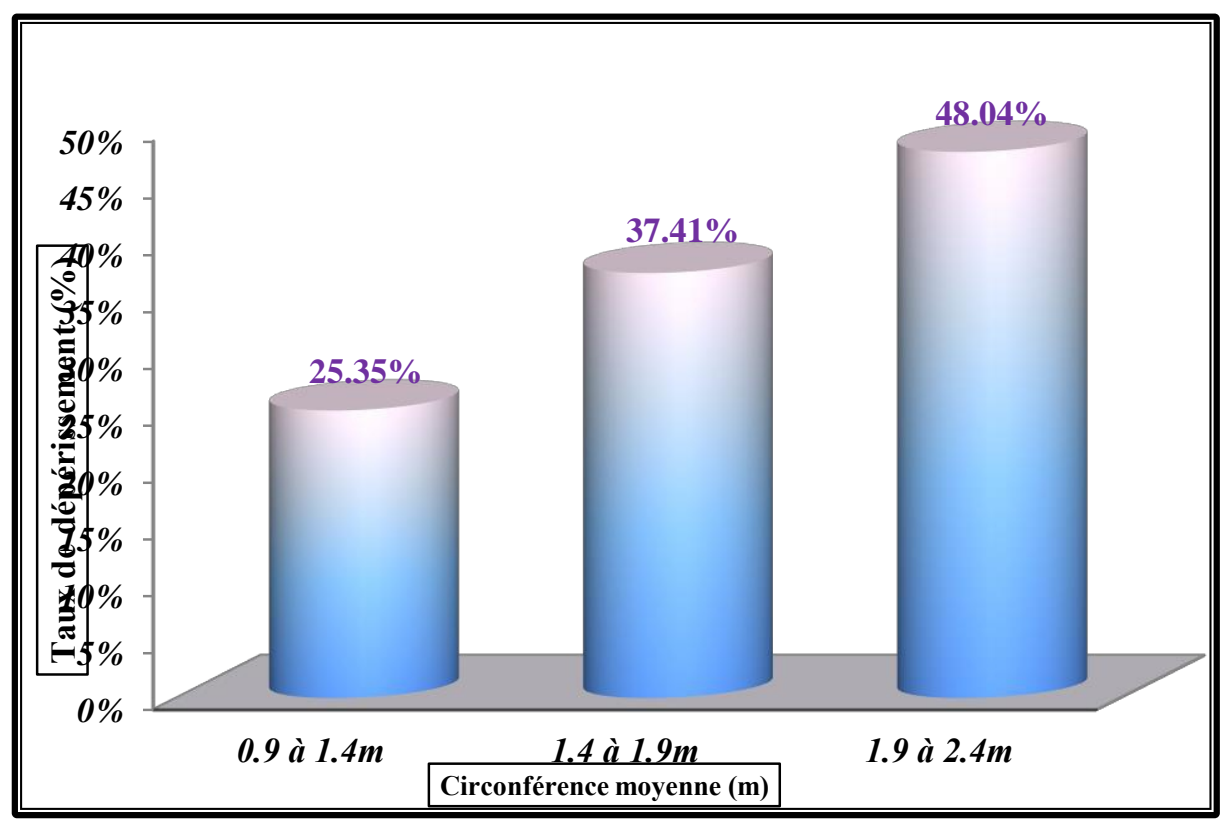

Figure 4 : Taux de dépérissement à travers la circonférence moyenne

\section{Conclusion}

La cédraie de Theniet El Had, est l'une des plus belles cédraies de l'Algérie. D'une altitude variant entre 1277 et $1786 \mathrm{~m}$, elle est soumise à l'étage bioclimatique sub-humide à hiver froid.

A travers l'installation de trente placettes circulaires de 10 ares, 507 arbres de cèdre ont été recensés dont 159 y sont dépéris soit un taux de $31 \%$.

L'étude phytosymptomatologique des arbres de cèdre à travers le recensement des maladies du feuillage et les dégâts sur les houppiers exprime une situation inquiétante sur le devenir de la cédraie, d'où les dommages des aiguilles sont évaluée à $34 \%$ et les dommages sur les houppiers sont de l'ordre de $30 \%$ des peuplements recensés. 
L'approche statistique employée pour l'étude de la relation entre le dépérissement et les paramètres écodendrométriques est l'analyse de la variance au seuil de signification de $95 \%$ qui est un test d'hypothèse de type paramétrique étudiant les relations de causalité entre une variable qualitative dite facteur et une variable quantitative mesurée. Les résultats obtenus révèlent que :

- La relation dépérissement-Facteurs stationnels ne présente aucune différence significative ;

- La relation dépérissement-facteurs édaphiques ne présente aucune différence significative, résultats déjà obtenus par les auteurs montrant que le cèdre est indifférent à la nature lithologique et aux propriétés chimiques du sol ;

- La relation dépérissement-variables dendrométriques liées à la fertilité ne présente aucune différence significative, un résultat confirmant celles des facteurs écologiques ;

$\mathrm{La}$ relation dépérissement-variables dendrométriques liées à la compétition ne présente aucune différence significative à l'exception de la circonférence moyenne qui présente une corrélation positive avec l'évolution du dépérissement.

Les peuplements de cèdre inventoriés se caractérisent par un une grande variabilité dendrométrique d'une placette à l'autre, situation compromettante, conséquente de l'issue naturelle de la cédraie, érigée en parc national et non soumise aux actions sylvicoles.

Ces observations confirment la nécessité d'établir un plan de gestion par une équipe pluridisciplinaire prenant en considération la compétition comme facteur prédisposant dans le processus du dépérissement qui entraine l'affaiblissement de la vigueur des arbres.

\section{References:}

1. Alteyrac, J. (2005). Influence de la densité de peuplement et de la hauteur dans l'arbre sur les propriétés physico-mécaniques du bois d'épinette noire (Piceamariana (mill.). Thèse Doctorat. Univ. LAVAL. QUEBEC. 136p.

2. Anonyme. (1984). Etude et projet pour la mise en valeur des terres, aménagement des forêts et des parcs nationaux dans le massif de l'Ouarsenis. Vol. 19. Lescomplekt. Bulgarie. 169p.

3. O.N.F, Office National des forêts. (2010). Guide de gestion des forêts en crise sanitaire. Paris, $100 \mathrm{p}$.

4. Bellefleur, P., Villeneuve, M. (1984). Evolution des taux de croissance juvénile d'espèces forestières sous trois régimes 
expérimentaux de compétition. Ann. Sci. Forest. Vol. 41, n 4 . pp 427-438.

5. Benabid, A. (1994). Biogéographie, phytosociologie et phytodynamique des cédraies de l'Atlas Cedrus atlantica (Manetti). Ann. Rech. For. Maroc. T 27. pp 61-76.

6. Benmouffok, A. (1994). Approche écopédologique dans les formations à Cedrus atlantica "Cas du massif du Djurdjura, Algérie". Ann. Rech. For. Maroc. T 27. pp 205-217.

7. Boudy, P. (1950). Economie forestière nord-africaine. Tome 02. Monographie et traitements des essences forestières. Larose. Paris. 878p.

8. Chbouki, N. (1994). Une synthèse dendroclimatique du cèdre de l'Atlas. Ann. Rech. For. Maroc. T 27. pp 33-59.

9. Dagnelie, P. (1994). Théorie et méthodes statistiques applications agronomiques : les méthodes de l'inférence statistique. 2ème édition. Les Presses agronomiques de Gembloux. Vol. II. 463p.

10. Décourt, N. (1967). Le douglas dans le nord-est du massif central : Tables de production provisoires. Ann. Sci. Forest, Vol. 24, n ${ }^{\circ}$. pp 45-84.

11. Deleuze, C., Blaudez, D., Herve, J.C. (1996). Ajustement d'un modèle hauteur-circonférence

12. Demarteau, M., Francois, L., Cheddadi, R., Roche, E. (2007). Réponses de Cedrus atlantica aux changements climatiques passés et futurs. Geo-Eco-Trop. n³1. pp 105-146.

13. Drénou, C. (2006). Les racines: face cachée des arbres. IDF. Paris. $335 \mathrm{p}$.

14. Guehl, J.M. (1984). Dynamique de l'eau dans le sol en forêt tropicale humide guyanaise. Influence de la couverture pédologique. Ann. Sci. Forest. Vol. 41, n². pp 195-236.

15. Haddar, L. (2007). Analyse qualitative et quantitative de l'entomofaune de la cédraie du parc national de Chréa (Blida).These.magi, INA, El-Harrach. Alger, 114p.

16. Le Goff, N., Levy, G. (1984). Productivité du frêne (Fraxinus excelsior L.) en région Nord- Picardie. B. - Etude des relations entre la productivité et les conditions de milieu. Ann. Sci. Forest. Vol. 41, $\mathrm{n}^{\circ} 2$. pp 135-170.

17. Lecomte, B. (2007). Le cèdre de l'Atlas en Languedoc-Roussillon. Forêt-entreprise, $\mathrm{n}^{\circ} 174$. pp 51-54.

18. Mathieu, C. Pieltain, F. (2003). Analyse chimique des sols : Méthodes choisies. TEC \& DOC. Paris. 387p.

19. Palm, R. (1977). Dendrométrie. Polycopié. INA. Alger. 112p. 
20. Parde, J. Bouchon, J. (1988). Dendrométrie. 2ème éd. ENGREF. Nancy. 328p.

21. Riou-Nivert, P. (2005). Les résineux : Ecologie et pathologie. Forêt Privée Française. $447 \mathrm{p}$.

22. Ripert, C. Boisseau, B. (1994). Ecologie et croissance du cèdre de l'atlas en Provence. Ann. Rech. For. Maroc. T 27. pp 155-171.

23. Rondeux, J. (1993). La mesure des arbres et des peuplements forestiers. Les presses agronomiques de Gembloux. 521p.

24. Rondeux, J. Thibaut, A. Claessens, H. (1995). Comment estimer la productivité des peuplements d'Epicéa commun (Picea abies(L.) Karst.) en Ardenne ?. Fiche technique $\mathrm{n}^{\circ} 8$, gestion et économie forestières section écologie. Faculté des Sciences agronomiques de Gembloux. 2p.

25. Seigue, A. (1985). La forêt circumméditerranéenne et ses problèmes. Maisonneuve \& Larose. 502p.

26. Toth, J. (1970). Plus que centenaire et plein d'avenir : le cèdre en France. Rev. For. Fr. Vol. XXII, n 03. pp 355-364. 